\title{
Neuroendoscopic lavage for the management of posthemorrhagic hydrocephalus in preterm infants: safety, effectivity, and lessons learned
}

\author{
Jorge Tirado-Caballero, MD, ${ }^{1}$ Mónica Rivero-Garvia, MD, PhD, ${ }^{1}$ Francisco Arteaga-Romero, MD, ${ }^{1}$ \\ Jorge Herreria-Franco, MD, ${ }^{1}$ Ángel Lozano-Gonzalez, MD, ${ }^{1}$ and Javier Marquez-Rivas, MD, PhD ${ }^{1,2}$
}

${ }^{1}$ Neurosurgery Service Virgen del Rocío, University Hospital, Seville; and ${ }^{2}$ Group of Applied Neuroscience, Biomedicine Institute of Seville, Spain

OBJECTIVE Treatment of posthemorrhagic hydrocephalus in preterm infants is a matter of debate among pediatric neurosurgeons. Neuroendoscopic lavage (NEL) has been proposed as a suitable technique for the management of this pathology. The authors present their experience with 46 patients treated with NEL after germinal matrix hemorrhage, describe their technique, and analyze the outcomes.

METHODS A retrospective analysis of patients affected by grade III or IV intraventricular hemorrhage (IVH) treated with NEL was performed. Nonmodifiable risk factors such as gestational age, weight at birth, modified Papile grade, and intercurrent diseases were reviewed. Safety analysis was performed, evaluating the incidence of postoperative complications. Effectivity was measured using radiological (frontal horn index and white matter injury), CSF biochemical (proteins, blood, and cellularity), and clinical variables. Permanent shunt rate and shunt survival were analyzed. The motor outcome was measured using the Gross Motor Function Classification System (GMFCS) at 18-24 months, and the neurocognitive outcome was evaluated according to the grade of adaptation to schooling.

RESULTS Forty-six patients met the inclusion criteria. Patients were treated with a single NEL in 21 cases, 2 lavages in 13 cases, and 3 lavages in 12 cases. The mean gestational age at birth was 30.04 weeks, and the mean weight at birth was $1671.86 \mathrm{~g}$. Hyaline membrane disease was described in $4.8 \%$ of the cases, hematological sepsis in $43.47 \%$, persistent ductus arteriosus in $23.9 \%$, and necrotizing enterocolitis in $10.9 \%$ of the cases. Modified Papile grade III and IV IVHs were observed in $60.9 \%$ and $39.1 \%$ of the cases, respectively. Postoperative infection was diagnosed in 10 of the 46 cases, CSF leak in 6, and rebleeding in 3. The mean frontal horn index decreased from $49.54 \mathrm{~mm}$ to $45.50 \mathrm{~mm}$ after NEL. No white matter injury was observed in 18 of 46 patients, focal injury was described in 13 patients, and diffuse bilateral white matter injury was observed in 15 patients. All CSF biochemical levels improved after lavage. The shunt rate was $58.7 \%$, and shunt survival at 1 year was $50 \%$. GMFCS grades I, II, III, IV, and V were observed in $44.74 \%, 21.05 \%$, $2.63 \%, 15.79 \%$, and $15.79 \%$ of patients, respectively. Good neurocognitive results were observed in $53.3 \%$ of the cases. CONCLUSIONS Neuroendoscopic lavage was a suitable alternative for the management of IVH in preterm infants in our series. Good motor and neurocognitive results were achieved by this technique, and the permanent shunt rate was reduced compared with historical controls.

https://thejns.org/doi/abs/10.3171/2020.2.PEDS2037

KEYWORDS intraventricular hemorrhage; preterm; germinal matrix; neuroendoscopy; ventricular lavage; hydrocephalus; congenital

I NTRAVENTRICULAR hemorrhage (IVH) is a serious disease associated with high morbidity and neurological impairment in preterm infants. ${ }^{1,2}$ Up to $25 \%-30 \%$ of preterm infants can suffer from $\mathrm{IVH}^{3}$ and 7\%-9\% will develop a high-grade IVH (grade III or IV). Also, up to $50 \%$ of patients with high-grade IVH will require neurosurgical treatment for secondary hydrocephalus. ${ }^{4}$

Several surgical approaches have been proposed for this complex entity, including external ventricular drainage ${ }^{5}$ ventriculosubgaleal shunt (VSGS); ${ }^{6,7}$ subcutaneous

ABBREVIATIONS DRIFT = drainage, irrigation, and fibrinolytic therapy; FHI = frontal horn index; GMFCS = Gross Motor Function Classification System; IVH = intraventricular hemorrhage; NEL = neuroendoscopic lavage; VAD = ventricular access device; VPS = ventriculoperitoneal shunt; VSGS = ventriculosubgaleal shunt. SUBMITTED January 13, 2020. ACCEPTED February 27, 2020.

INCLUDE WHEN CITING Published online May 15, 2020; DOI: 10.3171/2020.2.PEDS2037. 
reservoir access; or drainage, irrigation, and fibrinolytic therapy (DRIFT). ${ }^{8}$

Neuroendoscopic lavage (NEL) has also been described as a suitable technique for the management of this pathology. ${ }^{9,10}$ The main role of this procedure is similar to that of DRIFT: to achieve the removal of intraventricular blood clots to prevent secondary brain damage and control hydrocephalus. ${ }^{11}$ In addition, NEL has been associated with a lower rate of permanent shunting and longer shunt survival.

We report our series of 46 patients affected by germinal matrix hemorrhage treated with NEL. The surgical technique is described in detail, and its safety and effectiveness are analyzed.

\section{Methods}

We report our retrospective analysis of a unicentric series from Hospital Virgen del Rocío, Seville, Spain. Inclusion criteria were met by patients affected by hydrocephalus due to grade III or IV germinal matrix hemorrhage treated by the NEL method. The surgical procedure was performed only in patients who showed progressive hydrocephalus on radiological studies and when the patients were clinically stable. Patients with a poor prognosis because of uncontrollable hemodynamic instability due to premature birth were excluded from surgical treatment.

\section{Surgical Procedure}

Under general anesthesia, the patient was positioned supine on the operating table. A low-dose CT scan was used to plan the frontal precoronal approach. A rigid $0^{\circ}$ MINOP neuroendoscopy system (Aesculap) was introduced in the frontal horn of the lateral ventricle with a greater amount of blood (Fig. 1A). The trajectory of the endoscope to the ventricle was guided by transfontanellar ultrasonography. Once the endoscope was located inside the ventricular system, a CSF sample was taken for biochemical analysis, and continuous irrigation began. Usually, 2000-2500 mL of Ringer/Plasma-Lyte solution was used for irrigation. Lavage was started in the frontal horn and extended to the body and occipital horn of the ipsilateral ventricle (Fig. 1B). A ventricular drain was eventually used for irrigating and removing distant clots during the procedure. Once the ipsilateral ventricle was cleared, the endoscope was brought closer to the foramen of Monro to complete endoscopic third ventricle lavage (Fig. 1C).

After the third ventricle lavage, septostomy was performed to clean the contralateral ventricle. The endoscope was introduced through septostomy, and lavage of the contralateral frontal horn and body of the ventricle was performed (Fig. 1D). Again, a ventricular drain was inserted adjacent to the endoscope and used for irrigation of the contralateral occipital horn and removal of the distant blood clots. Irrigation continued until CSF was cleared in the entire ventricular system (Fig. 1E). Finally, hemostatic matrix (Floseal, Baxter) was placed at the intraventricular location of the germinal matrix hemorrhage (Fig. 1F). The corticectomy was sealed after lavage with 2 layers of Hemopatch (Baxter); the first was rolled up inside the ventricular track and the second over the pia mater surface.
No subcutaneous reservoirs or external ventricular drains were left after lavage. A video of our technique is provided (Video 1).

VIDEO 1. Surgical technique for performing NEL. The endoscope is introduced inside the ventricular system and lavage is done using a Ringer solution. After ipsilateral lavage, a septostomy is performed for contralateral endoscopic lavage. Lavage continues until the CSF is clear in both lateral ventricles and the third ventricle. Copyright Jorge Tirado-Caballero. Published with permission. Click here to view.

\section{Re-Lavage Criteria}

The criteria for repeating the lavage were the progression of hydrocephalus as observed on ultrasonography or CT scanning and the persistence of blood clots in the ventricular system as determined on imaging. We considered that IVH was not controlled under these circumstances and that a higher level of CSF clearance could be achieved to reduce the damage caused by the hemorrhage and improve the hydrocephalus control.

\section{Ventriculoperitoneal Shunt Insertion Criteria}

A ventriculoperitoneal shunt (VPS) was placed when hydrocephalus progression was observed on CT scanning or ultrasonography and the CSF was clear, or no blood clots were seen during the previous NEL. Proximal catheters were implanted occipitally, and programmable differential pressure valves were used in all patients. A minimum weight of $1500 \mathrm{~g}$ was required for this procedure.

\section{Nonmodifiable Risk Factors}

Nonmodifiable risk factors at birth, such as gestational age and weight at birth, were reviewed. Intercurrent diseases such as hyaline membrane disease, necrotizing enterocolitis, persistent ductus arteriosus, and hematological sepsis (described as positive blood cultures) were also evaluated.

The radiological severity of IVH was described according to the modified Papile classification. ${ }^{6}$ The grades of this scale are as follows: grade I, confined germinal matrix hemorrhage; grade II, $<50 \%$ of ventricular filling by the IVH; grade III, $>50 \%$ of ventricular filling by the IVH, leading to ventricular dilation; and grade IV, periventricular hemorrhage or infarction observed on radiological studies. ${ }^{6}$ All patients treated in this series had grade III or grade IV IVH. Patients affected by grade I or II IVH did not develop hydrocephalus and did not need surgical treatment and thus are not included in this study.

\section{Safety Analysis}

Postoperative complications, including infection, CSF leak, and postoperative rebleeding, were described and analyzed. Infection diagnosis was considered when CSF cultures were positive. Rebleeding was considered when the intraventricular blood volume increased in the first 72 hours after lavage.

Associations between the incidence of these complications and the epidemiological, medical, and surgical conditions were reviewed. Statistical analysis for safety results was performed using IBM SPSS (version 22, IBM 

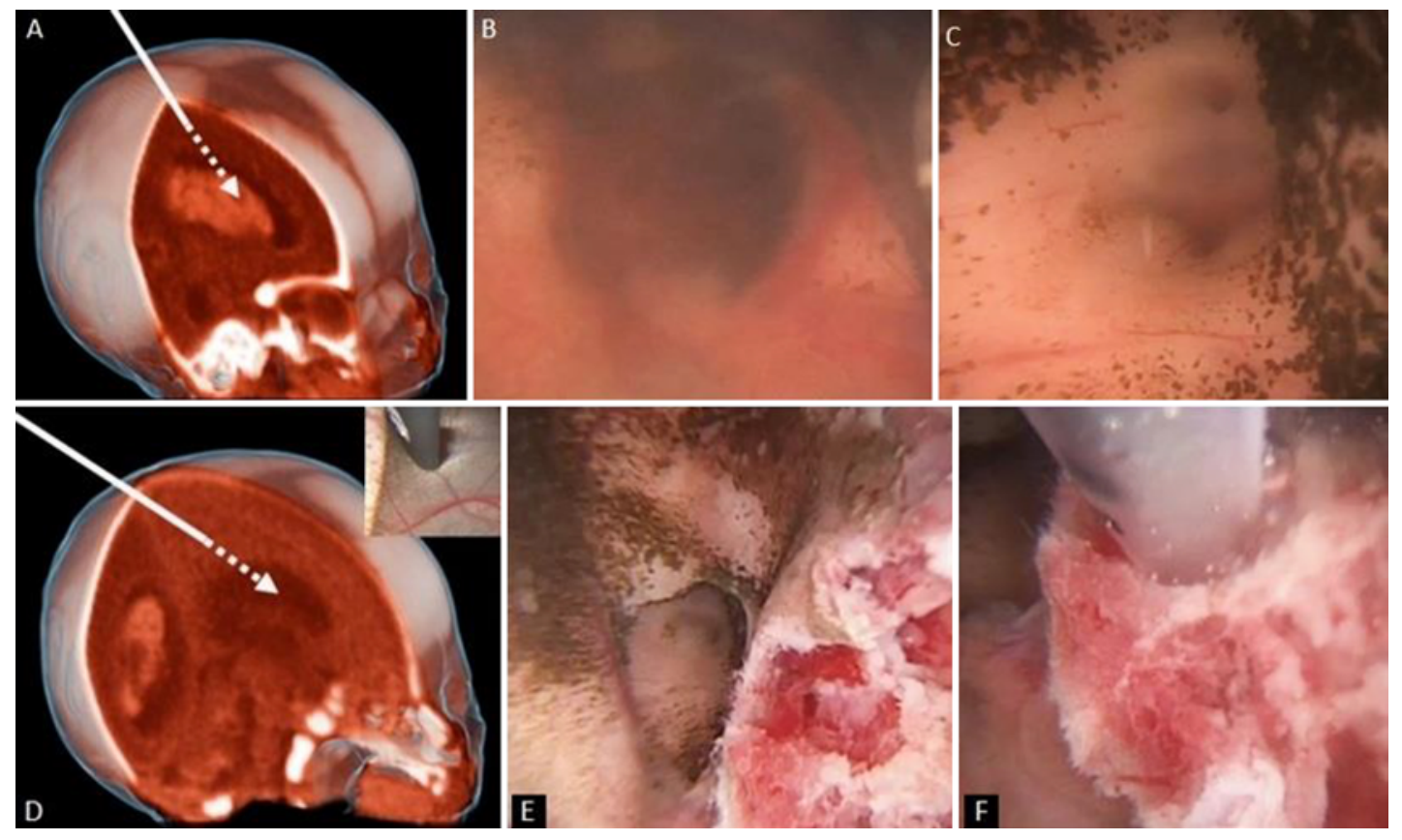

FIG. 1. A: 3D CT scan. Endoscopic trajectory to the frontal horn of the lateral ventricle. B: Endoscopic view of the frontal horn before starting irrigation. A blurry view (as shown) is common at this phase of surgery. C: Endoscopic view of the third ventricle before starting endoscopic lavage. The ependymal surface can be observed fully covered by blood clots. D: Endoscopic trajectory to the frontal horn of the contralateral ventricle. Inset: The endoscopic view of the septum pellucidum being opened to perform contralateral lavage. E: Final view of the ventricular system after the first lavage. Clear CSF was achieved, and the paraventricular structures are now identified. F: Hemostatic matrix is placed at the intraventricular location of the germinal matrix bleeding. Figure is available in color online only.

Corp.) relating weight at birth, gestational age, modified Papile grade, number of endoscopic lavages, and days until the first lavage with the incidence of these complications. Multivariate regression was performed, and $\mathrm{p}<0.05$ was considered statistically significant.

\section{Effectivity Analysis}

Effectivity in this study was assessed by radiological, biochemical, CSF shunting, and neurological outcome variables.

\section{Radiological Analysis}

The frontal horn index (FHI) before and after lavage procedures was measured and reviewed. Development of white matter injury (periventricular leukomalacia/white matter infarction or ischemia) in the 1st year was also reviewed. White matter injury was classified into 3 grades according to its extension: grade 1 , no injury; grade 2 , focal cystic injury; and grade 3 , diffuse bilateral white matter injury (Fig. 2).

\section{Biochemical Analysis}

CSF biochemical analysis was performed before and after the NELs. CSF proteins (mg/dL), blood count (blood cells $/ \mathrm{mL}$ ), and cellularity count (cells $/ \mu \mathrm{L}$ ) were analyzed. The first CSF sample was taken when the endoscope was introduced inside the ventricular system, prior to the beginning of continuous irrigation. A second CSF sample was taken by ventricular puncture only in cases of sus- pected CSF infection, a second NEL, or for the insertion of a VPS. This was done to avoid the potential risks associated with repeated ventricular punctures. Samples were only considered for postoperative analysis when the time between the NEL and the next sample was less than 1 week.

\section{CSF Shunting Analysis}

The need for a permanent shunt after NEL, the age at shunt insertion, and the time to first shunt malfunction/ shunt survival (in months) at 3 months, 6 months, and 1 year were reviewed. Statistical analysis for CSF shunting results was performed using IBM SPSS (version 22) relating modified Papile grade, the number of endoscopic lavages, infection, and CSF biochemical levels (proteins, cellularity, and blood) with the need for a permanent shunt and shunt survival. Multivariate regression was performed, and $p<0.05$ was considered statistically significant.

\section{Clinical Outcome Analysis}

Mortality in the 1st year was analyzed. Pathologies related to these deaths were also reviewed.

Neurological motor impairment was analyzed using the Gross Motor Function Classification System (GMFCS) at 18-24 months (Table 1). ${ }^{12}$ Patients were assessed by an expert physician in the Cerebral Palsy Rehabilitation Unit in our center. Good neurological motor outcome was defined as GMFCS grade I or II, and a bad neurological outcome was considered as GMFCS grades III-V. 

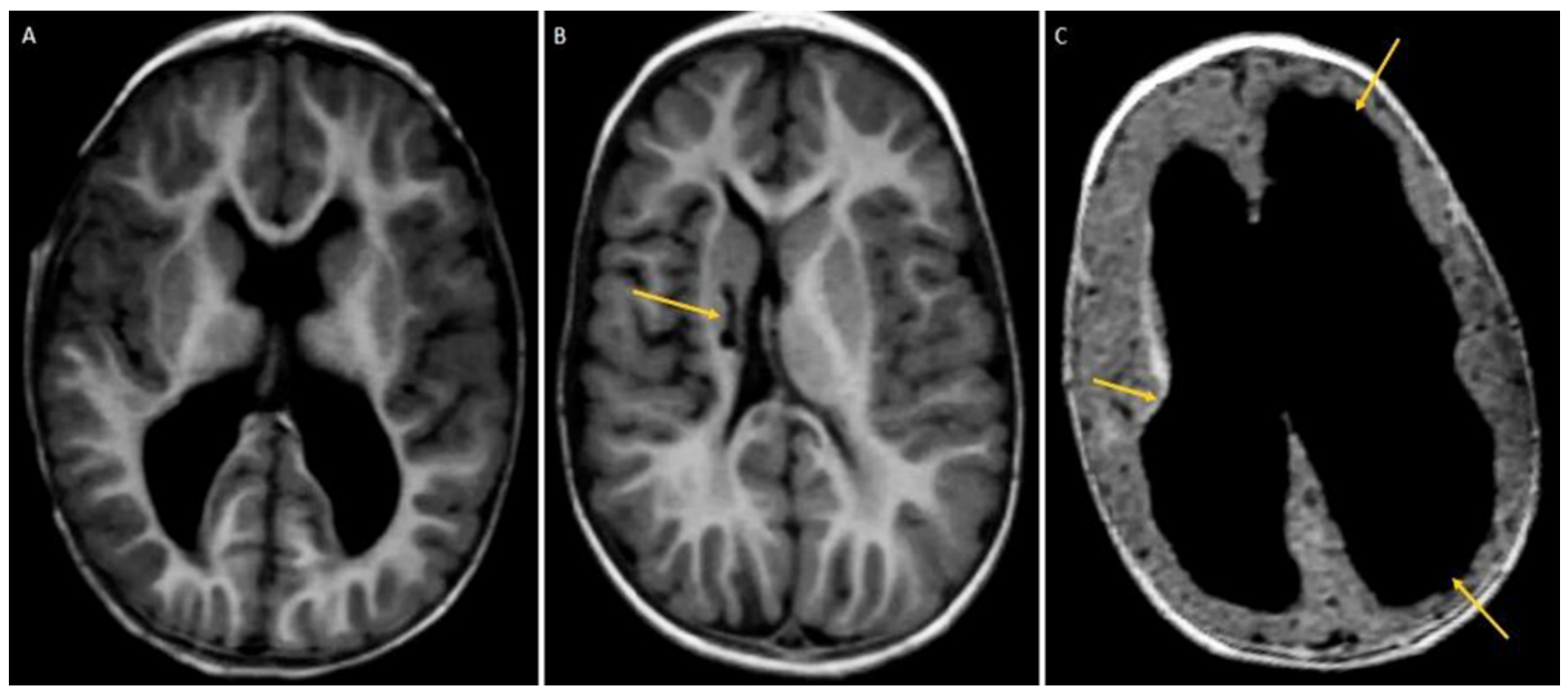

FIG. 2. Grades of white matter injury. A: No injury. B: Focal unilateral cystic injury (yellow arrow). C: Diffuse bilateral white matter injury (yellow arrows). Figure is available in color online only.

Neurocognitive results were established according to the adaptation of the patient to schooling. According to our grading system, patients in grade 1 attend regular school at the proper school year without needing extra support, special speech therapy, or psychotherapy. Those in grade 2 attend regular school at the proper school year but need extra support classes or speech therapy. Patients in grade 3 attend regular school but not at the proper school year and need curricular adaptation. Patients in grade 4 are not able to attend regular school and must go to specific classrooms for personal education. Patients younger than 3 years who did not attend school were not included in this cognitive analysis. A good neurocognitive result was considered as grade 1 and 2 patients on this scale (patients who attend their proper school year at the time of analysis). A poor neurocognitive result was considered to be grade 3 or 4 .

Statistical analysis for motor and cognitive results was performed using IBM SPSS (version 22). The Student t-

TABLE 1. GMFCS extended and revised

\begin{tabular}{cc}
\hline GMFCS grade & Definition \\
\hline I & Walk w/o limitations \\
\hline III & Able to walk but w/ some limitations \\
\hline IV & Able to walk but a handheld mobility device is needed \\
\hline Adapted for children <2 yrs & Self-mobility w/ limitations; may use powered mobility \\
\hline I & Transported in a manual wheelchair \\
\hline II & $\begin{array}{c}\text { Infant moves in \& out of sitting \& floor sit w/ both hands free to manipulate } \\
\text { objects; crawls on hands \& knees, pulls to stand \& takes steps holding to } \\
\text { furniture; walks btwn } 18 \text { mos \& 2 years w/o need of any assistive mobility } \\
\text { device }\end{array}$ \\
\hline III & $\begin{array}{c}\text { Infant maintains floor sitting but may need to use hands for support to main- } \\
\text { tain balance; may pull to stand \& take steps holding to furniture }\end{array}$ \\
\hline IV & $\begin{array}{c}\text { Infant maintains floor sitting when the low back is supported; rolls \& creeps } \\
\text { over stomach }\end{array}$ \\
\hline V & $\begin{array}{c}\text { Infant has head control but trunk support is required for floor sitting; can roll } \\
\text { to supine \& may roll to prone }\end{array}$ \\
\hline & $\begin{array}{c}\text { Physical impairment limits voluntary control of movement; infant is unable to } \\
\text { maintain antigravity head \& trunk postures in prone \& sitting; requires adult } \\
\text { assistance to roll }\end{array}$ \\
\hline
\end{tabular}


test was used for quantitative analysis of CSF biochemical values and radiological FHI. A univariate regression model was performed relating every single variable with motor and neurocognitive outcomes. Multivariate linear regression was performed for all variables that showed statistical significance $(\mathrm{p}<0.05)$ in the univariate regression model.

\section{Results}

Forty-six patients met the inclusion criteria. The first patient was treated in May 2012. The last patient included in this study was treated in January 2019. A single NEL was performed in 21 of 46 patients, 2 lavages in 13 patients, and 3 lavages in 12 patients. The mean time to the first lavage was 20.85 days (SD 17.43 days). The mean follow-up in our series was 46.91 months (SD 21.69 months).

\section{Nonmodifiable Risk Factors}

The mean gestational age at birth in our series was 30.04 weeks (SD 4.79 weeks). The mean weight at birth was $1671.86 \mathrm{~g}$ (SD $827.84 \mathrm{~g}$ ). The most common intercurrent diseases diagnosed in this series were hyaline membrane disease in $22(4.8 \%)$ of 46 patients, hematological sepsis in 20 patients $(43.48 \%)$, persistent ductus arteriosus in 11 patients (23.9\%), and necrotizing enterocolitis in 5 patients $(10.9 \%)$. Regarding radiological grade of the patients treated with NEL, modified Papile grade III IVH was described in 28 patients (60.9\%), and modified Papile grade IV was observed in 18 patients (39.1\%).

\section{Safety Analysis}

Infection was diagnosed in 10 (21.7\%) of the 46 patients. The incidence of infection directly increased according to the number of lavages and was diagnosed in $1(4.76 \%)$ of 21 patients treated with a single NEL, $2(15.38 \%)$ of 13 patients treated with 2 lavages, and $7(58.33 \%)$ of 12 patients treated with a third endoscopic lavage. This relationship was also statistically significant $(\mathrm{p}<0.05)$ in multivariate analysis. No other risk factors for infection were related to a higher incidence of CSF infection (Fig. 3).

CSF leak occurred in $6(13.04 \%)$ of 46 patients. All leaks were repaired with nonabsorbable monofilament stitches and a mild compressive bandage. The incidence of CSF leak was $1(4.76 \%)$ of 21 patients treated with a single NEL, $2(15.38 \%)$ of 13 patients treated with 2 lavages, and $3(25 \%)$ of 12 patients treated with 3 lavages. The incidence of CSF leak increased with the number of lavages, but this relationship was not statistically significant in the multivariate analysis $(p=0.215)$. No other risk factors described above were related to a higher incidence of CSF leak. Rebleeding occurred in $3(6.52 \%)$ of 46 patients. No risk factors described above were related to the incidence of rebleeding.

\section{Effectivity Analysis \\ Radiological Results}

The mean FHI decreased from $49.54 \mathrm{~mm}$ (SD 0.89 $\mathrm{mm}$ ) prior to lavage to $45.50 \mathrm{~mm}$ (SD $0.98 \mathrm{~mm}$ ) after NEL. This difference was statistically significant $(\mathrm{p}<$ 0.05 , Student t-test). Regarding white matter injury, no in-

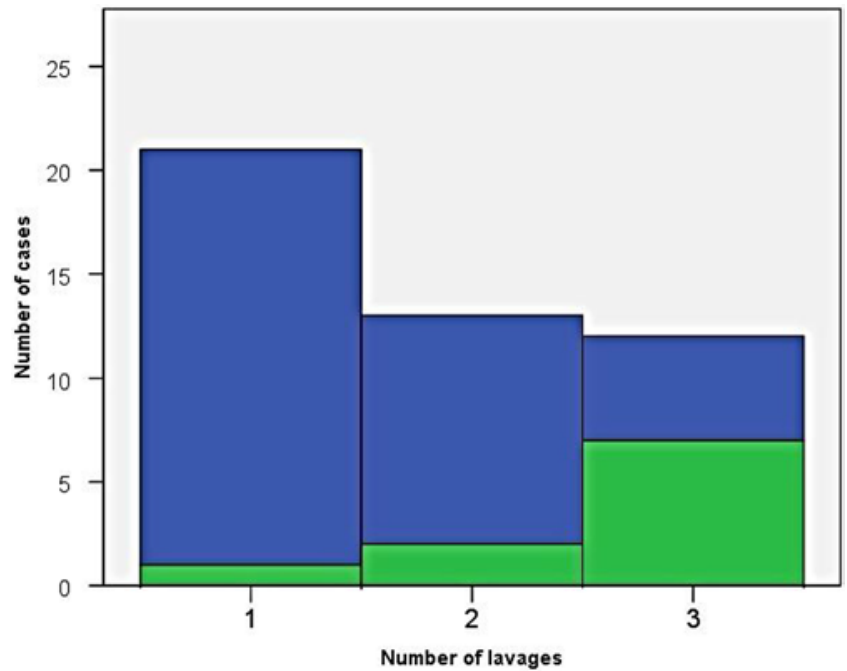

FIG. 3. Blue: noninfected patients; green: infected patients. Only 1 $(4.76 \%)$ of 21 patients was infected after the first lavage, $2(15.38 \%)$ of 13 patients after the second lavage, and $7(58.33 \%)$ of 12 patients after a third endoscopic lavage. The relationship between the number of lavages and the incidence of infection was statistically significant in our series $(p<0.05)$. Figure is available in color online only.

jury was observed in 18 (39.13\%) of 46 patients, minimal focal injury was described in 13 patients $(28.26 \%)$, and diffuse bilateral white matter injury was found in 15 patients $(32.6 \%)$.

\section{Biochemical Results}

Postoperative CSF samples were taken in 27 of 46 patients. The mean CSF protein level was $2.15 \mathrm{mg} / \mathrm{dL}$ (SD $0.99 \mathrm{mg} / \mathrm{dL}$ ) before NEL and $1.58 \mathrm{mg} / \mathrm{dL}$ (SD $0.60 \mathrm{mg} / \mathrm{dL}$ ) after lavage. The mean CSF cellularity was 276.65 cells/ $\mu \mathrm{L}$ (SD 563.11 cells $/ \mu \mathrm{L}$ ), which decreased to 32.33 cells/ $\mu \mathrm{L}$ (SD 40.70 cells $/ \mu \mathrm{L}$ ) after the procedure. The mean blood count in the CSF was 32,888 cells/mL (SD 56,619 cells $/ \mathrm{mL}$ ), decreasing to 5174.6 cells $/ \mathrm{mL}$ (SD 8160.31 cells/ $\mathrm{mL}$ ) after surgery. All differences were statistically significant ( $\mathrm{p}<0.05$, Student t-test).

\section{CSF Shunting Results}

After NEL, the shunt dependency rate was 58.7\% (27/ 46 patients). Shunt insertion was performed when the patients had a mean corrected age of 3.32 months (SD 1.89 months) corresponding to 45.01 gestational weeks (SD 16.79 weeks). VPS placement was necessary for $33 \%$ of the patients treated with a single lavage, $84.6 \%$ of the patients treated with 2 lavages, and $75 \%$ of the patients treated with 3 lavages. The mean shunt survival in our series was 19.53 months (SD 25.25 months). Shunt survival at 3 months was $69.23 \%$, which decreased to $52.17 \%$ at 6 months and to 50\% at the 1-year follow-up (Fig. 4).

Statistical analysis did not show a significant relationship between the modified Papile IVH grade, incidence of infection, number of endoscopic lavages, or CSF biochemical levels, and the need for permanent shunt or shunt survival. 


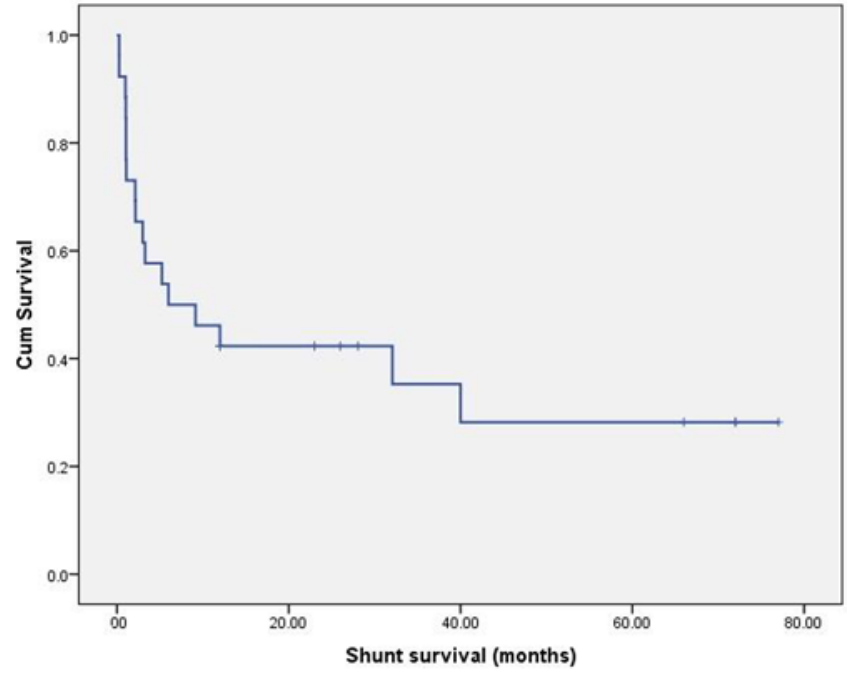

FIG. 4. Kaplan-Meier analysis showing shunt survival in our series with time (months). Fifty percent of the shunts failed in the 1st year. After 18 months of follow-up, the shunt malfunction rate remained more stable. Figure is available in color online only.

\section{Clinical Outcome Results}

Three $(6.52 \%)$ of the 46 patients died within the 1st year. None of these deaths were related to postoperative complications of the procedure, but instead to respiratory, cardiac, and infectious problems associated with the premature birth.

At the time of clinical outcome analysis, 5 patients had not yet reached 18 months old (minimum age for outcome evaluation). The 3 patients who died in the 1 st year were also excluded. Therefore, motor and neurocognitive outcomes were only analyzed in 38 of 46 patients who met the criteria.
Neurological motor function was established using the GMFCS scale at 18-24 months. Using this scale, 17 (44.74\%) of 38 patients showed GMFCS grade I. GMFCS grade II was observed in 8 patients $(21.05 \%)$, grade III in 1 patient (2.63\%), grade IV in 6 patients (15.79\%), and grade $\mathrm{V}$ in 6 patients (15.78\%) (Fig. 5 left). A good neurological motor (GMFCS grade I or II) function was observed in 25 patients $(65.79 \%)$. However, 13 patients $(34.21 \%)$ had a poor neurological motor outcome (GMFCS grade III, IV, or V).

In univariate analysis, patients younger than 30 weeks and weighing less than $1200 \mathrm{~g}$ showed a higher incidence of GMFCS grades III-V. However, patients older than 30 weeks of gestational age and with a higher weight showed better motor outcomes. These two variables were statistically significant ( $p<0.05$; Fig. 6). However, none of the analyzed intercurrent diseases worsened the neurological motor outcome in GMFCS. No statistical differences were observed between patients with intercurrent diseases and those without $(\mathrm{p}>0.05)$.

Regarding radiological studies, the neurological motor outcome as measured by GMFCS grade at 18-24 months was worse in modified Papile grade IV hemorrhages than in grade III in univariate analysis ( $p<0.05$; Fig. 7 left). The grade of white matter injury was highly related to motor outcome ( $\mathrm{p}<0.01 ; \mathrm{R}=0.735)$. Accordingly, diffuse white matter injury was related to worse motor outcome and higher levels of long-term spasticity in our series (Fig. 7 right). Otherwise, the preoperative and postoperative FHI did not show a statistical relationship with the motor outcome.

Postoperative complications such as CSF leak and rebleeding were not associated with a worse motor outcome at $18-24$ months in our series $(p>0.05)$. However, the incidence of infection was associated with a worse outcome ( $\mathrm{p}<0.009 ; \mathrm{R}=0.420$ ).

No biochemical variables (preoperative/postoperative CSF proteins, cellularity, or blood count) were related to
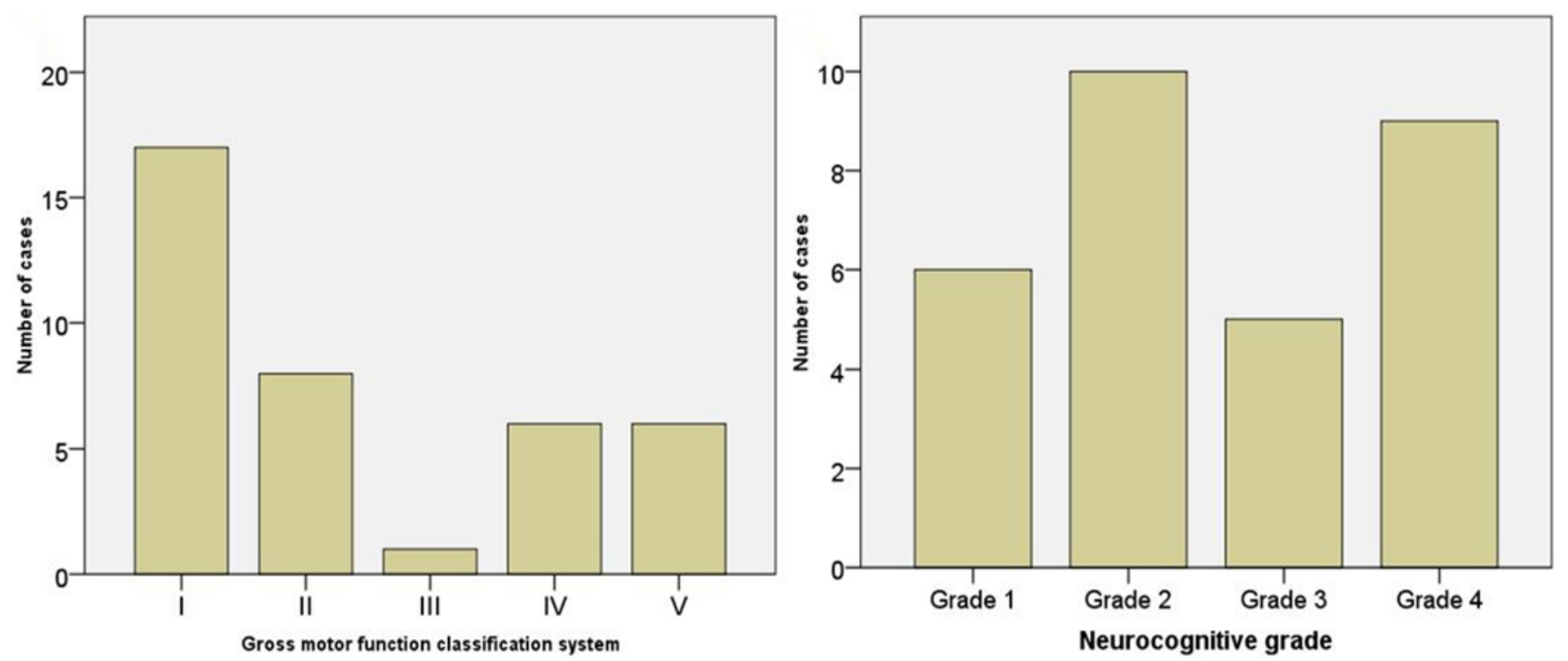

FIG. 5. Left: Motor outcome in our series, measured by the GMFCS at 18-24 months. Right: Neurocognitive development in our series, according to the adaptation to the schooling of the patient. Figure is available in color online only. 

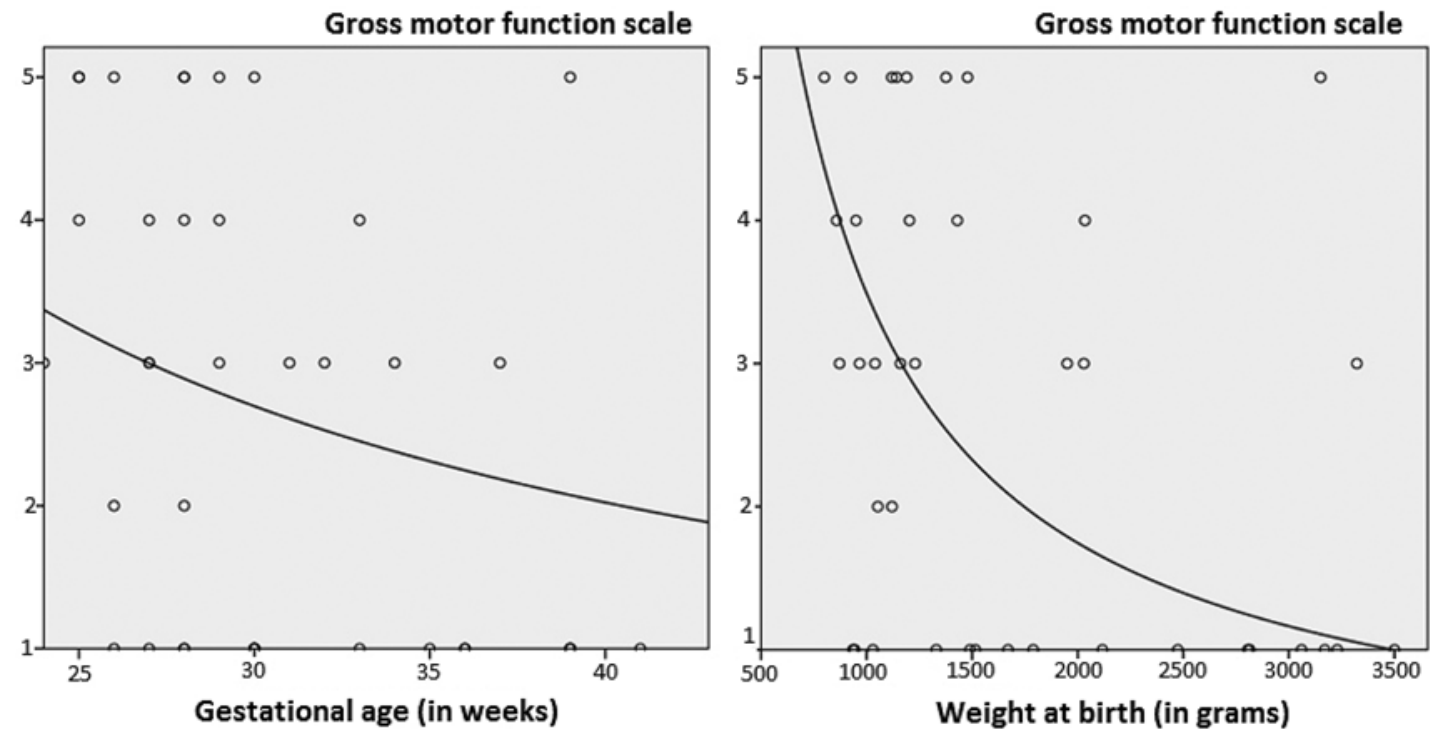

FIG. 6. Linear correlation between gestational age (in weeks, left) and weight at birth (in grams, right) with GMFCS grade. Patients with a gestational age $>30$ weeks and weight at birth $>1500 \mathrm{~g}$ have a better motor outcome.

the motor outcome in univariate analysis $(\mathrm{p}>0.05)$. Also, the need for a permanent CSF shunt, gestational age at shunt insertion, and shunt survival were not related to GMFCS results in univariate analysis $(\mathrm{p}>0.05)$. However, a greater number of lavages was related to a worse outcome $(\mathrm{p}<0.05 ; \mathrm{R}=0.469)$.

Multivariate regression analysis was performed for variables that showed statistical differences in univariate analysis (gestational age at birth, weight at birth, modified Papile grade, white matter injury grade, infection, and number of lavages). Only the white matter injury grade was statistically significant in this analysis $(\mathrm{p}<0.05)$. The modified Papile grade was also close to statistical significance in multivariate analysis $(p=0.053)$, but did not reach the established limit $(\mathrm{p}<0.05)$ for type 1 error.

Neurocognitive analysis was performed in 30 of 46 patients who met the criteria. Grade 1 was observed in 6 (20\%) of 30 patients, grade 2 in 10 patients (33.3\%), grade 3 in 5 patients $(16.7 \%)$, and grade 4 in 9 patients $(30 \%)$. Thus, a good neurocognitive outcome was described in $53.3 \%$ of the patients and a bad neurocognitive outcome in 46.7\% (Fig. 5 right). Also, GMFCS scores and neurocogni-
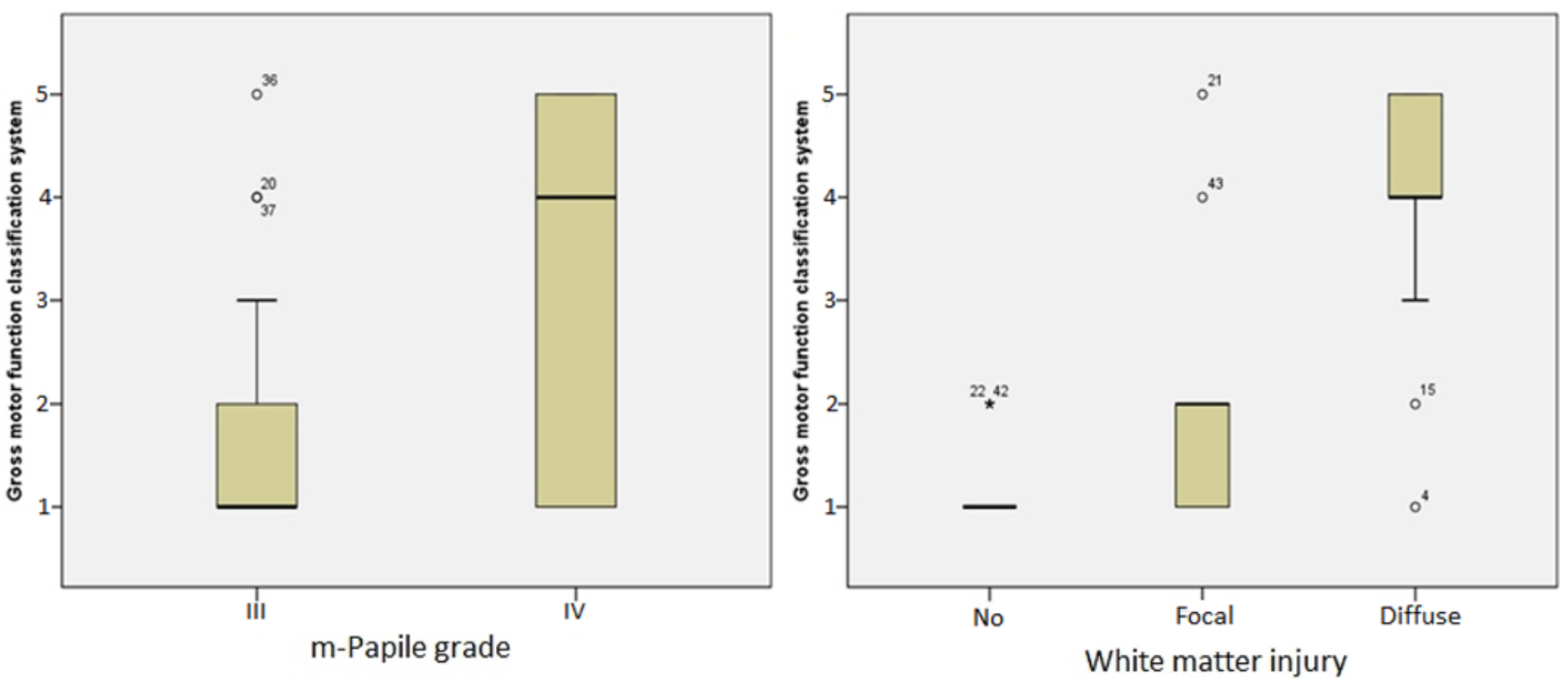

FIG. 7. Boxplots showing the relationship between GMFCS score at 18-24 months and modified Papile (m-Papile) grade (left) and the white matter injury (right). Grade IV IVH and diffuse white matter injury were the variables most related to motor outcome in our series. Numbers within the plots represent patient numbers. Circles indicate outliers. The star in the right panel indicates that only 2 patients were in GMFCS grade II. Figure is available in color online only. 
tive results were highly related in our series (Pearson $\mathrm{R}=$ $0.800)$.

Univariate analysis showed a significant relationship between poorer cognitive results and lower weight at birth $(\mathrm{p}=0.029 ; \mathrm{R}=0.399)$, lower gestational age at birth ( $\mathrm{p}$ $=0.011 ; \mathrm{R}=0.457)$, higher incidence of infection $(\mathrm{p}=$ $0.005 ; \mathrm{R}=0.497)$, modified Papile grade IV hemorrhage $(\mathrm{p}=0.003 ; \mathrm{R}=0.528)$, diffuse white matter injury $(\mathrm{p}=$ $0.001 ; \mathrm{R}=0.587)$, need for permanent shunt $(\mathrm{p}=0.023$; $R=0.415)$, and greater number of lavages $(p=0.002 ; R=$ $0.533)$. Multivariate regression analysis was performed for these variables, but none reached statistical significance.

\section{Discussion}

Surgical treatment of grade III and IV germinal matrix hemorrhages is still a matter of concern among pediatric neurosurgeons. The damage caused by both hydrocephalus and the hemorrhage itself is an important cause of neurological morbidity among preterm infants. ${ }^{1,13}$

Recent studies of the premature human brain described the importance of the generation and later migration of GABAergic interneurons from the germinal matrix to the cerebral cortex between weeks 25 and 34. This impairment in the neuronal generation and migration secondary to germinal matrix injury could explain the deleterious consequences for neurodevelopment in these children. ${ }^{14,15}$

The diversity of treatments that have been used for hydrocephalus control in these patients has deepened the controversy around the best management for this complex disease. ${ }^{16}$ Also, subsequent surgery for permanent CSF diversion has been described in $70 \%-75 \%$ of the cases, and short- and long-term complications related to these procedures have been widely reported in the literature. ${ }^{17,18}$

According to the literature, the infection rate for VSGS placement is approximately $9.2 \%-14 \%$; obstruction was described in $9.6 \%$ of patients and arrested hydrocephalus in $13.9 \%$, as well as a $12.1 \%$ mortality rate, a $12.1 \%$ revision rate, and a $58.7 \%$ rate of good neurodevelopmental outcome. ${ }^{19}$ A $6.5 \%-10 \%$ rate of CSF leakage in VSGSs was described by some authors. ${ }^{7}$ Also, in patients treated with VSGSs, a permanent shunt was necessary in $76.1 \%$ of the cases. ${ }^{20}$

The rate of infection for the ventricular access device (VAD) has been reported at $9.5 \%-20 \%$. The percentage of infection was directly related to the number of tappings. ${ }^{20}$ Obstruction of the device was observed in $7.3 \%$, arrested hydrocephalus in $17.5 \%$, and mortality was described in $15.3 \%$. A good neurodevelopmental outcome was observed in $50.1 \%$ of the cases. A permanent shunt in patients with a VAD was necessary in $77.3 \%$ of the cases. A CSF leak was observed in $22 \%$ of the cases in some series. A recent meta-analysis did not show significant differences between these VSGS and VAD procedures or in the safety or outcome results. ${ }^{19}$

DRIFT was described by Whitelaw et al. ${ }^{21}$ as another modality of treatment for this serious disease. These authors introduced 2 external ventricular drains inside the ventricular system and irrigated them with recombinant tissue plasminogen activator and saline solution. However, a $34 \%$ rebleeding rate was observed in this study, and recruitment was discontinued. ${ }^{8}$ Interestingly, the removal of the intraventricular hematoma described in DRIFT achieved better neurological and cognitive development 2 and 10 years after the procedure despite a high rate of early rebleeding. ${ }^{22}$

NEL offers a suitable alternative for the management of these patients. Continuous irrigation inside the ventricle with direct visualization of the blood clots prevents the described risks associated with the intraventricular infusion of recombinant tissue plasminogen activator in DRIFT. Also, this procedure achieves CSF clearance and reduces the presence of residual blood clots attached to the ependymal surface. With this technique, the number of degradation products that can cause delayed neuroinflammatory reactions over brain parenchyma was drastically reduced.

Complications related to NEL have been described. Schulz et al. found CSF leaks in $5.4 \%$ of patients after this procedure, and CSF infections were found in 3.6\% of patients, as diagnosed by positive cultures. ${ }^{10}$ Some differences regarding patient management can be observed between the Charité group and our group. ${ }^{9,10}$ Their authors described the insertion of a VAD after NEL, which was used to both relieve intracranial pressure and take CSF samples. In our series, no VAD was introduced after NEL. Also, the number of ventricular lavages differs between the series. In the Charite series, most of the patients were treated with a single NEL, and only 9 of them were treated with a second lavage. In our series, patients were treated with 2 and 3 lavages in $28.26 \%$ and $26.09 \%$ of the cases, respectively.

This issue could explain the main differences in the rate of complications between series. The infection rate, for example, increased with every lavage, from $4.76 \%$ in the first lavage to $58.33 \%$ after the third lavage. Also, CSF leakage $(13.4 \%)$ increased with the number of lavages from $4.76 \%$ to $25 \%$. Based on these findings, we consider that the decision of going beyond the second lavage must be made cautiously and only for selected cases, as we proved that there is not enough evidence to consider that a third lavage would improve the outcome or reduce the rate of permanent CSF shunting.

On the other hand, some interesting data were revealed after this study. Biochemical analysis, for example, showed improvement in CSF characteristics (proteins, blood, and cellularity) days after lavage. We hypothesize that this biochemical improvement could be the cause of the reduced number of patients who needed a permanent CSF shunt in our series (58.7\%). However, statistical analysis did not show a clear relationship between these variables. The small number of patients $(n=46)$ in this series could be the reason for this lack of statistical evidence. Thus, in our opinion, a larger series could be useful for correlating this biochemical CSF status with shunt dependency and shunt survival.

Schulz et al. found that $58 \%$ of patients needed a shunt in their NEL series and reported higher shunt survival compared with controls (64\% at 1 year of follow-up)., ${ }^{9,10,23}$ However, shunt survival results in our series were not as optimistic. Fifty percent of the patients had shunt malfunctions in the 1st year, so that this milestone could still be unknown.

Cerebral palsy is another matter of concern when 
studying this entity. According to some authors, the probability of suffering cerebral palsy increases with the grade of IVH..$^{1,24}$ Other authors have described the worsening of pure motor function, adaptative fine and gross motor function, and balance with each grade of IVH..$^{25,26}$ In this study, we described the correlation between modified Papile grade IV IVH and adverse neurological outcome in univariate analysis. This correlation was even stronger when analyzing the white matter injury caused by the IVH and hydrocephalus.

The outcome results in our series were promising compared with historical control groups. GMFCS grades I and II at $18-24$ months were described in $65.78 \%$ of patients. This means that patients can walk and control floor sitting. Also, neurocognitive results were very optimistic, with $53.3 \%$ of the patients adapting to school in their proper school year. These results are encouraging for the therapeutic effects of blood lavage to reduce neuroinflammation and improve the long-term outcome. Of course, this is a small group of patients compared with other studies, and these results must be confirmed in multicenter trials.

On this issue, a multicenter clinical trial for posthemorrhagic hydrocephalus management is being developed today in many pediatric neurosurgery units around the world. ${ }^{27}$ Published multicenter trials have not included NEL as a possible treatment for this pathology. We hope that the results of this trial enlighten and provide evidence to know the best management for this challenging disease. However, many of the risk factors related to unsatisfactory outcome in our series were nonmodifiable, such as gestational age at birth, weight at birth, or modified Papile grade. Therefore, the chance to improve the prognosis in these patients will be limited by these factors, and prevention in neonatal intensive care units is certainly mandatory.

\section{Conclusions}

Neuroendoscopic lavage was a suitable and feasible technique for the management of hydrocephalus after germinal matrix hemorrhage in our series. A lower permanent shunt rate and good neurological outcome in both motor and cognitive function were observed in our series. Further studies and randomized trials are necessary to compare the different treatment strategies and establish reliable scientific evidence in this controversial subject.

\section{References}

1. Mukerji A, Shah V, Shah PS. Periventricular/intraventricular hemorrhage and neurodevelopmental outcomes: a meta-analysis. Pediatrics. 2015;136(6):1132-1143.

2. Chan K, Ohlsson A, Synnes A, et al. Survival, morbidity, and resource use of infants of 25 weeks' gestational age or less. Am J Obstet Gynecol. 2001;185(1):220-226.

3. Heuchan AM, Evans N, Henderson Smart DJ, Simpson JM. Perinatal risk factors for major intraventricular haemorrhage in the Australian and New Zealand Neonatal Network, 199597. Arch Dis Child Fetal Neonatal Ed. 2002;86(2):F86-F90.

4. Christian EA, Melamed EF, Peck E, et al. Surgical management of hydrocephalus secondary to intraventricular hemorrhage in the preterm infant. J Neurosurg Pediatr. 2016;17(3):278-284.

5. Bassan H, Eshel R, Golan I, et al. Timing of external ventric- ular drainage and neurodevelopmental outcome in preterm infants with posthemorrhagic hydrocephalus. Eur J Paediatr Neurol. 2012;16(6):662-670.

6. Fulmer BB, Grabb PA, Oakes WJ, Mapstone TB. Neonatal ventriculosubgaleal shunts. Neurosurgery. 2000;47(1):80-84.

7. García-Méndez A, Álvarez-Vázquez L, Agustín-Aguilar F, et al. Ventriculo subgaleal shunt in hydrocephalus secondary to intraventricular hemorrhage in prematures. Article in Spanish. Gac Med Mex. 2014;150(suppl 3):279-281.

8. Whitelaw A, Pople I, Cherian S, et al. Phase 1 trial of prevention of hydrocephalus after intraventricular hemorrhage in newborn infants by drainage, irrigation, and fibrinolytic therapy. Pediatrics. 2003;111(4 pt 1):759-765.

9. D'Arcangues C, Schulz M, Bührer C, et al. Extended experience with neuroendoscopic lavage for posthemorrhagic hydrocephalus in neonates. World Neurosurg. 2018;116:e217e224.

10. Schulz M, Bührer C, Pohl-Schickinger A, et al. Neuroendoscopic lavage for the treatment of intraventricular hemorrhage and hydrocephalus in neonates. $J$ Neurosurg Pediatr. 2014;13(6):626-635.

11. Koschnitzky JE, Keep RF, Limbrick DD Jr, et al. Opportunities in posthemorrhagic hydrocephalus research: outcomes of the Hydrocephalus Association Posthemorrhagic Hydrocephalus Workshop. Fluids Barriers CNS. 2018;15(1):11.

12. Palisano R, Rosenbaum P, Walter S, et al. Development and reliability of a system to classify gross motor function in children with cerebral palsy. Dev Med Child Neurol. 1997;39(4):214-223.

13. Bolisetty S, Dhawan A, Abdel-Latif M, et al. Intraventricular hemorrhage and neurodevelopmental outcomes in extreme preterm infants. Pediatrics. 2014;133(1):55-62.

14. Bystron I, Blakemore C, Rakic P. Development of the human cerebral cortex: Boulder Committee revisited. Nat Rev Neurosci. 2008;9(2):110-122.

15. Xu G, Broadbelt KG, Haynes RL, et al. Late development of the GABAergic system in the human cerebral cortex and white matter. J Neuropathol Exp Neurol. 2011;70(10):841858.

16. Valdez Sandoval P, Hernández Rosales P, Quiñones Hernández DG, et al. Intraventricular hemorrhage and posthemorrhagic hydrocephalus in preterm infants: diagnosis, classification, and treatment options. Childs Nerv Syst. 2019;35(6):917-927.

17. Brouwer AJ, Groenendaal F, van den Hoogen A, et al. Incidence of infections of ventricular reservoirs in the treatment of post-haemorrhagic ventricular dilatation: a retrospective study (1992-2003). Arch Dis Child Fetal Neonatal Ed. 2007;92(1):F41-F43.

18. Spader HS, Hertzler DA, Kestle JR, Riva-Cambrin J. Risk factors for infection and the effect of an institutional shunt protocol on the incidence of ventricular access device infections in preterm infants. $J$ Neurosurg Pediatr. 2015;15(2):156-160.

19. Badhiwala JH, Hong CJ, Nassiri F, et al. Treatment of posthemorrhagic ventricular dilation in preterm infants: a systematic review and meta-analysis of outcomes and complications. J Neurosurg Pediatr. 2015;16(5):545-555.

20. Wang JY, Amin AG, Jallo GI, Ahn ES. Ventricular reservoir versus ventriculosubgaleal shunt for posthemorrhagic hydrocephalus in preterm infants: infection risks and ventriculoperitoneal shunt rate. J Neurosurg Pediatr. 2014;14(5):447454.

21. Whitelaw A, Jary S, Kmita G, et al. Randomized trial of drainage, irrigation and fibrinolytic therapy for premature infants with posthemorrhagic ventricular dilatation: developmental outcome at 2 years. Pediatrics. 2010;125(4):e852e858.

22. Luyt K, Jary S, Lea C, et al. Ten-year follow-up of a ran- 
domised trial of drainage, irrigation and fibrinolytic therapy (DRIFT) in infants with post-haemorrhagic ventricular dilatation. Health Technol Assess. 2019;23(4):1-116.

23. Wellons JC III, Shannon CN, Holubkov R, et al. Shunting outcomes in posthemorrhagic hydrocephalus: results of a Hydrocephalus Clinical Research Network prospective cohort study. J Neurosurg Pediatr. 2017;20(1):19-29.

24. Larroque B, Marret S, Ancel PY, et al. White matter damage and intraventricular hemorrhage in very preterm infants: the EPIPAGE study. J Pediatr. 2003;143(4):477-483.

25. Bassan H, Limperopoulos C, Visconti K, et al. Neurodevelopmental outcome in survivors of periventricular hemorrhagic infarction. Pediatrics. 2007;120(4):785-792.

26. Pierrat V, Duquennoy C, van Haastert IC, et al. Ultrasound diagnosis and neurodevelopmental outcome of localised and extensive cystic periventricular leucomalacia. Arch Dis Child Fetal Neonatal Ed. 2001;84(3):F151-F156.

27. Thomale UW, Cinalli G, Kulkarni AV, et al. TROPHY registry study design: a prospective, international multicenter study for the surgical treatment of posthemorrhagic hydrocephalus in neonates. Childs Nerv Syst. 2019;35(4):613-619.

\section{Disclosures}

The authors report no conflict of interest concerning the materi- als or methods used in this study or the findings specified in this paper.

\section{Author Contributions}

Conception and design: Tirado-Caballero. Acquisition of data: Tirado-Caballero. Analysis and interpretation of data: TiradoCaballero. Drafting the article: Tirado-Caballero. Critically revising the article: Rivero-Garvia, Arteaga-Romero, Herreria-Franco, Lozano-Gonzalez, Marquez-Rivas. Reviewed submitted version of manuscript: Arteaga-Romero, Marquez-Rivas. Approved the final version of the manuscript on behalf of all authors: TiradoCaballero. Statistical analysis: Tirado-Caballero. Administrative/ technical/material support: Tirado-Caballero, Herreria-Franco, Lozano-Gonzalez. Study supervision: Marquez-Rivas.

\section{Supplemental Information \\ Videos}

Video 1. https://vimeo.com/396429249.

\section{Correspondence}

Jorge Tirado-Caballero: University Hospital, Seville, Spain. jtiradocaballero@gmail.com. 